\title{
A Dynamic System Approach to the Characterization of the Response Behavior of a Class of Materials with the Inclusion of Time-History Effects
}

\author{
Y.M. Haddad and P. Yu \\ Department of Mechanical Engineering, University of Ottawa \\ Ottawa, Canada K1N 6 N5
}

\begin{abstract}
A new dynamic system identification method is proposed for the determination of the characteristic function of a class of linear rheological material systems, whereby the latter is considered as a dynamic system. In this, a dynamic system identification method is presented for the determination of the relaxation or creep function of the material from dynamic experimental measurements. First, the relation between the relaxation or creep function and the frequency response function of the system is established by assuming a model of rational function of polynomials for the frequency response function. Second, a discrete-time system analysis is introduced to identify the order and the parameters of the model. The results show that the proposed model is efficient and, meantime, easy to use.
\end{abstract}

\section{INTRODUCTION}

A fundamental task of most physical sciences is the establishment of mathematical models for the analysis, prediction and control of physical processes. Such models may be obtained by adopting, for instance, one of the following two approaches:

1. Physical reasoning by observing the behavior of the physical process.

2. Mathematical modeling that would be based on the analysis of experimental data pertaining to the system.

The first approach is based mainly on the analysis of the basic constitutive properties of the system. The second approach, however, does not concern itself about the type of the physical system it is dealing with. Instead, it analyses and establishes a model from the experimental input and output data and/or signals concerning the system.

In this paper, a new dynamic system identification method is proposed for the determination of the characteristic system function of a linear rheological material from a pertaining dynamic experimental system analysis. In this context, a model of rational function of polynomials for the so-called "transfer response 
function" is assumed. Here, a discrete-time system analysis method is first introduced to identify the order and parameters of the model. Then, the characteristic function of the system is obtained by using an inverse integral method. The numerical scheme and pertaining examples for testing the model are presented. It is concluded that the proposed procedure, although powerful, is easy to use and the model is accurate and efficient.

\section{PROCEDURES, RESULTS AND DISCUSSION}

\section{The Model}

Symbolically, the response behaviour of a homogeneous physical system can be expressed as

$$
y(t)=\Omega(x, t) x(t)
$$

Where $x(t)$ is the input, $y(t)$ is the corresponding response and $\Omega($.$) represents the system. The latter is$ referred to as the system operator. Mathematically, a linear system would satisfy the following criterion:

$$
\begin{aligned}
& y_{1}(t)=\Omega x_{1}(t) \\
& y_{2}(t)=\Omega x_{2}(t)
\end{aligned}
$$

and if $\alpha_{1}$ and $\alpha_{2}$ are constants, then

$$
\alpha_{1} y_{1}(t)+\alpha_{2} y_{2}(t)=\Omega\left[\alpha_{1} x_{1}(t)+\alpha_{2} x_{2}(t)\right]
$$

Usually, we are interested in obtaining explicit relationships between the input and output variables of a system. For a rheological system with a time-dependent history, such response relation may be expressed, in terms of the hereditary integral of the system (see Haddad [1995]) as

$$
y(t)=\int_{-\infty}^{\infty} x(t-\xi) h(\xi) d \xi
$$

In equation (4) above, $h(\xi)$ is referred to as the "system characteristic function". For a causal system, we have

$$
y(t)=\int_{0}^{\infty} x(t-\xi) h(\xi) d \xi
$$


In Eqn. (5), if the input $x(t)$ and the system characteristic function $h(t)$ are known, the output of the system may be obtained by a convenient numerical algorithm. In this paper, however, our problem is an inverse one, that is, if the output $y(t)$ and input $x(t)$ have been obtained from experiments, we would like to identify the system, that is, to determine the characteristic function $h(t)$ of the system. It is the so-called "system identification problem". Thus, a new approach to the solution of such a problem is introduced in this paper. We seek first a representation of the constitutive relationship in frequency domain. If we apply Laplace transform to Eqn. (5), we have the following relation

$$
Y(s)=H(s) X(s)
$$

where the theorem of Laplace transform of the convolution of two signals has been used (e.g., Fodor [1965]). In equation (6), $H(s)$ is referred to as the "transfer function" of the continuous-time system. It is expressed as

$$
H(s)=\int_{0}^{\infty} h(t) e^{-s t} d t
$$

Now, we assume the following rational function for $H(s)$

$$
H(s)=\frac{O(s)}{P(s)}=\frac{b_{1} s^{p-1}+b_{2} s^{p-2}+\ldots+b_{p}}{s^{p}+a_{1} s^{p-1}+\ldots+a_{p}}
$$

in which,

$$
\begin{aligned}
& Q(s)=b_{1} s^{p^{-1}}+b_{2} s^{p-2}+\ldots+b_{p-1} s+b_{p} \\
& P(s)=s^{p}+a_{1} s^{p-1}+\ldots+a_{p-1} s+a_{p}
\end{aligned}
$$

where $b_{1}, b_{2}, \ldots, b_{p}, a_{1}, a_{2}, \ldots, a_{p}$ are constant parameters, and $p$ is the order of the polynomial. The order of the polynomial $Q(s)$ above, is taken to be one less than the order of the polynomial $P(s)$; see $\mathrm{Yu}$ and Haddad [1995]. On the basis of the assumption of Eqn. (8), equation (6) becomes

$$
Y(s)=\frac{Q(s)}{P(s)} X(s)
$$

which can be rewritten as

$$
P(s) Y(s)=Q(s) X(s)
$$

Taking the inverse Laplace transform of Eqn. (11), we obtain, in view of (9), the corresponding model in time-domain, i.e., 


$$
\begin{gathered}
\quad \frac{d^{p}}{d t^{p}} y(t)+a_{1} \frac{d^{p-1}}{d t^{p-1}} y(t)+\ldots+a_{p} \nu(t) \\
=b_{1} \frac{d^{p-1}}{d t^{p-1}} x(t)+\dot{b}_{i} \frac{d^{p-2}}{d t^{p-2}} r(t)+\ldots+h_{p} x(t)
\end{gathered}
$$

Assuming that the $\mathrm{p}$-th order equation

$$
\xi^{p}+a_{1} \xi^{p-1}+\ldots+a_{p-1} \xi+a_{p}=0
$$

has the roots

$$
\xi_{1}, \xi_{2}, \ldots, \xi_{p}
$$

then, with reference to $(8)$, we can write the transfer function $H(s)$ in the following partial fraction form

$$
\begin{aligned}
H(s) & =\frac{b_{1} s^{p-1}+\xi_{2} s^{p-2}+\ldots+h_{p}}{\left(s-\xi_{1}\right)\left(s-\xi_{2}\right) \ldots\left(s-\xi_{p}\right)} \\
& =\frac{A_{1}}{s-\xi_{1}}+\frac{A_{2}}{s-\xi_{2}}+\ldots+\frac{A_{p}}{s-\xi_{p}} \\
& =\sum_{m=1}^{p} \frac{A_{m}}{s-\xi_{m}}
\end{aligned}
$$

where $A_{m}(m=1,2, \ldots, p)$ can be calculated by

$$
\begin{aligned}
A_{m} & =\lim _{s \rightarrow \xi_{m}} H(s)\left(s-\xi_{m}\right) \\
& =\frac{b_{1} \xi_{m}^{p-1}+b_{2} \xi_{m}^{p-2}+\ldots+b_{p-1} \xi_{m}+b_{p}}{\prod_{\substack{k=1 \\
k * m}}^{p}\left(\xi_{m}-\xi_{k}\right)}
\end{aligned}
$$

By taking the inverse Laplace transform of Eqn. (15) and noting that the inverse Laplace transform of $l /(s-\xi)$ is $e^{\xi}$, we can obtain the time-domain model for the system transfer function $h(t)$ as

$$
\begin{gathered}
h(t)=\sum_{m=1}^{p} A_{m} e^{\xi_{m} t} \\
(m=1,2, \ldots, p)
\end{gathered}
$$




\section{Determination of the Model}

In the previous subsection, the model for the transfer function was established. In this subsection, however, we discuss the determination of the parameters pertaining to the model from the dynamic experimental measurements. For this purpose, assuming, in the dynamic experiment, one can obtain two general input and output discrete-time series, respectively, as

$$
\begin{aligned}
& x\left(t_{0}\right), x\left(t_{1}\right), \ldots, x\left(t_{N-1}\right), x\left(t_{N}\right) \\
& y\left(t_{0}\right), y\left(t_{1}\right), \ldots, y\left(t_{N-1}\right), y\left(t_{N}\right)
\end{aligned}
$$

Now, one has to analyze the two discrete-time series of Eqn. (18) in order to determine the parameters $A_{m}$, $\xi_{m}(m=1,2, \ldots, p)$ of Eqn. (17). For this purpose, we introduce the following discrete-time system, in view of the continuous-time differential model, Eqn. (12).

$$
\begin{gathered}
y_{k}+\beta_{1} y_{k-1}+\ldots+\beta_{p-1} y_{k-p+1}+\beta_{p} y_{k-p} \\
=\alpha_{0} x_{k}+\alpha_{1} s_{k-1}+\ldots+\alpha_{p-1} x_{k-p+1} \\
(k=0,1,2, \ldots)
\end{gathered}
$$

where $\alpha_{p}, \alpha_{l}, \ldots, \alpha_{p-l}, \beta_{1}, \beta_{2}, \ldots, \beta_{p}$, and $p$ are constant parameters. Let

$$
D y_{k}=y_{k-1}
$$

and

$$
\begin{aligned}
& \varphi(D)=1+\underline{1}_{i} D+\ldots+\beta_{p} D^{p} \\
& \theta(D)=\alpha_{0}+\alpha_{1} D+\ldots+\alpha_{p-1} D^{p-1}
\end{aligned}
$$

Then, Eqn. (19) can be written as

$$
\varphi(D) y_{k}-\theta(D) x_{k}
$$

Representing the z-transforms of $\left\{y_{k}\right\}$ and $\left\{x_{k}\right\}$ by $Y(z)$ and $\left.X z\right)$ respectively, and taking the z-transform of Eqn. (22), one has

$$
\varphi\left(z^{-1}\right) Y(z)=\theta\left(z^{-1}\right) X(z)
$$

or

$$
Y(z)=\frac{q_{z}\left(z^{-1}\right)}{\varphi\left(z^{-1}\right)} X(z)=H_{d}(z) X(z)
$$


where $H_{d}(z)$ is called the "transfer function" of the discrete-time system. In view of (21) and (24), $H_{d}(z)$ acquires the form:

$$
H_{\alpha}(z)=\frac{\theta\left(z^{-1}\right)}{\varphi(z-1)}=\frac{\alpha_{0}+\alpha_{1} z^{-1}+\ldots+\alpha_{p-1} z^{-(p-1)}}{1+\beta_{i} z^{-1}+\ldots+\beta_{p-1} z^{-(p-1)}+\beta_{p} z^{-1}}
$$

Assume that the equation

$$
\varphi\left(\lambda^{-1}\right)=1+\beta_{1} \lambda^{-1}+\ldots+\beta_{p} \lambda^{-p}=0
$$

has roots $\lambda_{1}, \lambda_{2}, \ldots \lambda_{p}$, then, Eqn. (25) can be written as

$$
\begin{aligned}
H_{d}(z) & =\frac{\alpha_{0}+\alpha_{1} z^{-1}+\ldots+\alpha_{p-\mathrm{i}} z^{-p+1}}{\left(1-\lambda_{\mathrm{i}} z^{-1}\right)\left(1-\lambda_{2} z^{-1}\right) \ldots\left(1-\lambda_{p} z^{-1}\right)} \\
& =\frac{B_{1}}{1-\lambda_{1} z^{-1}}+\frac{B_{2}}{1-\lambda_{2} z^{-i}}+\ldots+\frac{B_{p}}{1-\lambda_{p} z^{-1}} \\
& =\sum_{m=1}^{p} \frac{B_{m}}{1-\lambda_{m} z^{-1}}
\end{aligned}
$$

where $B_{m}(m=1,2, \ldots, p)$ are calculated by

$$
\begin{aligned}
B_{m} & =\lim _{z \rightarrow \lambda_{m}} H_{d}(z)\left(1-\lambda_{m} z^{-1}\right) \\
& =\frac{\sim_{0}^{\sim}+\sim_{1}^{-1} \lambda_{m}^{-1}+\ldots+\alpha_{p-1} \lambda_{m}^{-p+1}}{\prod_{\substack{k=1 \\
k \neq m}}^{p}\left(1-\lambda_{k} \lambda_{m}^{-1}\right)}
\end{aligned}
$$

Taking the inverse z-transform of Eqn. (27), one obtains the system characteristic series or weighting sequence of the discrete-time system:

$$
\begin{gathered}
h_{d}(k)=\sum_{m=1}^{p} B_{m} \lambda_{m}^{k} u(k) \\
(m=1,2, \ldots, p \text { and } k=0,1,2, \ldots)
\end{gathered}
$$

At this point, one has to establish a relation between the system characteristic function $h(t)$ of a continuoustime system and the system characteristic series $h_{d}(k)$ of the corresponding discrete-time system. Therefore, we approximate the integral representation of Eqn. (5) by 


$$
\begin{gathered}
y(\Delta T \cdot i)=\sum_{k=0}^{\infty} h(k \cdot \Delta T) x[\Delta T \cdot i-\Delta T \cdot k] \cdot \Delta T \\
=\sum_{k=0}^{\infty} h(k \cdot \Delta T) x[\Delta T \cdot(i-k)] \cdot \Delta T \\
(i=0,1,2, \ldots)
\end{gathered}
$$

By the convolution property of the z-transform, and taking the inverse z-transform of Eqn. (24), the relation between the input and output of the discrete-time system, in the discrete-time domain, can be expressed as

$$
\begin{gathered}
y_{i}=\sum_{k=0}^{\infty} h(k \cdot \Delta T) x_{i-k} \cdot \Delta T \\
(i, k=0,1,2, \ldots)
\end{gathered}
$$

By comparing equations (30) and (31), the following equation will approximately hold

$$
h(k \cdot \Delta) \Delta T=h_{d}(k)
$$

Meantime, one has

$$
\begin{aligned}
& A_{m}=\frac{1}{\Delta T} B_{m} \\
& \xi_{m}=\frac{1}{\Delta T} \ln \lambda_{m} \quad(m=1,2, \ldots p)
\end{aligned}
$$

To determine the parameters $\mathrm{B}_{\mathrm{m}}, \lambda_{\mathrm{m}}(\mathrm{m}=1,2, \ldots, \mathrm{p})$ for the discrete-time system, the parameters $\alpha_{0}, a_{l}$, $\ldots, a_{p}, \beta_{l}, \beta_{2}, \ldots, \beta_{p}$ in the model Eqn. (25) have to be determined first. For this purpose, one chooses arbitrarily values of an order $p$ and of parameters $\alpha_{k}, \alpha_{1}, \ldots, \alpha_{p}, \beta_{l}, \beta_{2}, \ldots, \beta_{p}$ of a discrete-time system. Designating the chosen parameters by an over " $\wedge$ " and substituting them into Eqn. (19), we have

$$
\begin{gathered}
y_{i}+\hat{\beta}_{1} y_{i-1}+\ldots+\hat{\beta}_{p} y_{i-p} \\
=\hat{\alpha}_{0} x_{i}+\hat{\alpha}_{1} x_{i-1}+\ldots+\hat{\alpha}_{p-1} x_{i-p+1}+e_{i} \\
(t=1,2, \ldots, N)
\end{gathered}
$$

where $e_{i}(i=1,2, \ldots, N)$ are the errors encountered due to the arbitrary choice of the values of the parameters $\alpha_{n,} \alpha_{l}, \ldots, \alpha_{p}, \beta_{l}, \beta_{2} \ldots, \beta_{p}$ and the order $p$. The errors $\mathrm{e}_{\mathrm{i}}(\mathrm{i}=1,2, \ldots, \mathrm{N})$ can be expressed as 


$$
\begin{gathered}
e_{i}=y_{i}-\left(-y_{i-1}, \ldots,-y_{i-p}, x_{i}, \ldots, x_{i-n+1}\right)\left\{\begin{array}{c}
\hat{\beta}_{1} \\
\vdots \\
\hat{\beta}_{p} \\
\hat{\alpha}_{0} \\
\hat{\alpha}_{1} \\
\vdots \\
\hat{\psi}_{\bar{p}}:
\end{array}\right\} \\
=y_{i}-\left\{w_{i}\right\}^{T}\{\hat{\beta}\} \\
(i=1,2, \ldots, N)
\end{gathered}
$$

where

$$
\begin{aligned}
& \left\{w_{t}\right\}^{T}=\left(-y_{i-1}, \ldots,-y_{i-p}, x_{i}, x_{i-1}, \ldots, x_{i-p+1}\right) \\
& \{\hat{\beta}\}^{T}=\left(\hat{\beta}_{1}, \ldots, \hat{\beta}_{p}, \hat{\alpha}_{0}, \hat{\alpha}_{1}, \ldots, \hat{\alpha}_{p-1}\right)
\end{aligned}
$$

and " $T$ " represents the transpose of a matrix.

By minimizing the sum of the square of the error, i.e.,

$$
\begin{aligned}
e^{2} & =\frac{1}{N} \sum_{i=D+1}^{N} e_{1}^{2} \\
& =\frac{1}{N} \sum_{i=D+1}^{N}\left(y_{1}-\left\{w_{i}\right\}^{T}\{\hat{\beta}\}\right)\left(y_{i}-\left\{w_{i}\right\}^{T}\{\hat{\beta}\}\right)
\end{aligned}
$$

one has

$$
\left(\frac{1}{N} \sum_{i=p-1}^{N}\left\{w_{i}\right\}\left\{w_{i}\right\}^{I}\right)\{\beta\}=\frac{1}{N} \sum_{i=p-1}^{N} y_{i}\left\{w_{i}\right\}
$$

Thus, for every choice of an order $p$, a corresponding $\{\hat{\beta}\}$ can be determined by Eqn. (38). Then, from Eqn. (37), the pertaining sum of the squares of the errors can, then, be calculated. The choice of the order $p$ of the discrete-time system can be made by requiring that it would result in a minimum sum of the squares of the errors.

\section{Numerical Examples}

To test the analytical model developed in the previous two subsections, a number of numerical 
illustrations are performed below. The formalism of these illustrations is outlined as follows:

1. For a given system, calculate the response of the system under certain dynamic loading by a numerical method (Runge-Kattaa Method, e.g. Morris [1983] is employed). Then, two discrete-time series (one is the input to the system and the other is its response) are obtained.

2. Assuming that no other knowledge about the system is given except the two discrete-time series of input and output, Eqn. (18), apply the proposed "Dynamic System Identification Method" to the two discretetime series. First, determine the parameters of the discrete-time system (DTS) function, then, determine the corresponding continuous system function.

\section{Example (1)}

Consider the system

$$
\ddot{y}+25 \dot{y}+100 y=100 \sin \left(t^{1.5}\right)
$$

with the input $x(t)$

$$
x(t)=100 \sin \left(0.5 t^{1.5}\right)
$$

The input $x(t)$ of the system is plotted in Figure 1, with $\Delta \mathrm{T}=0.01$. The parameters of this system are listed in Tables 1 and 2 below.

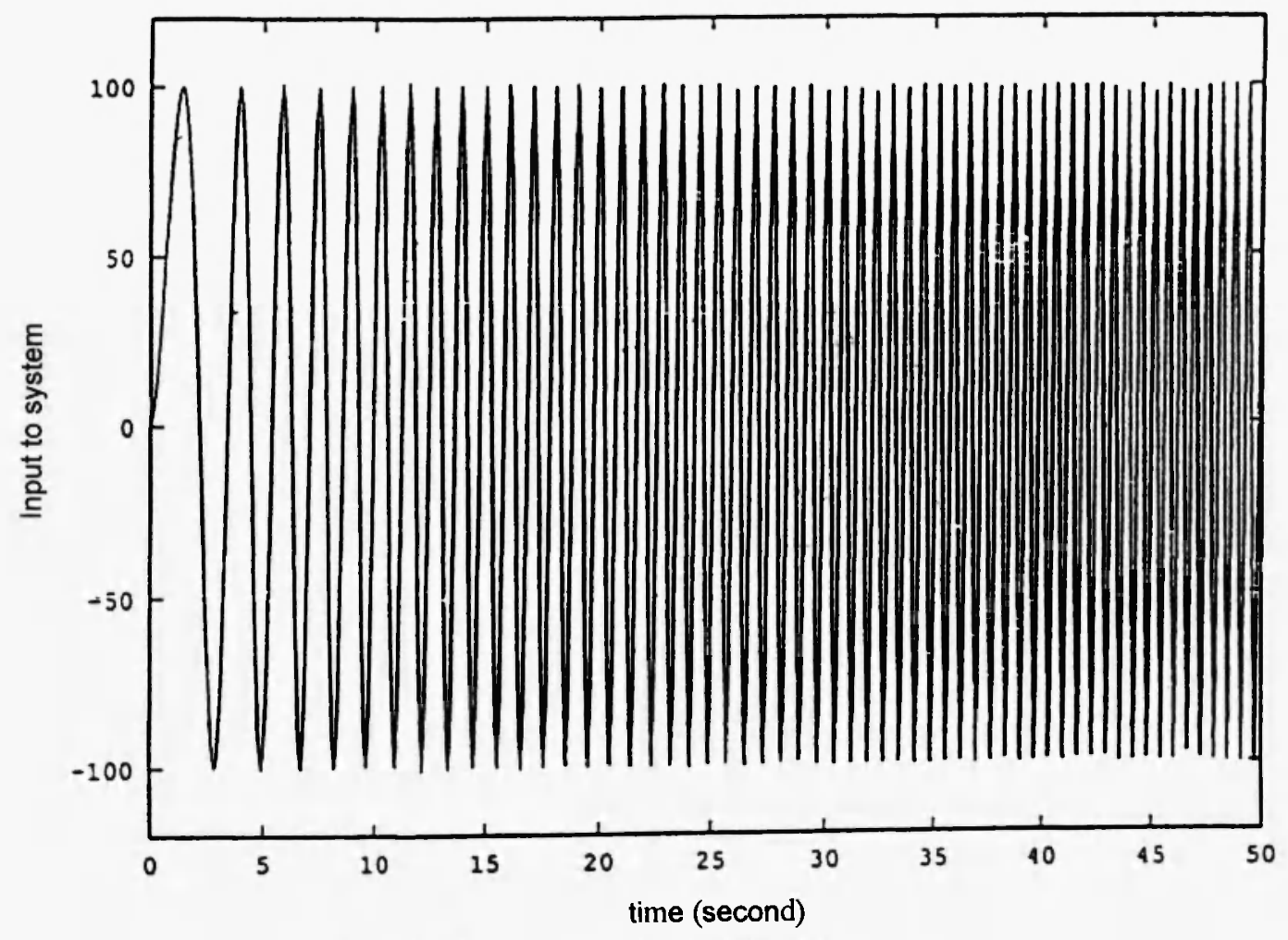

Fig. 1: Input $\mathrm{x}(\mathrm{t})=100 \sin \left(\mathrm{t}^{1.5}\right)$ with $\Delta \mathrm{T}=0.01$. 
Table 1

Parameters identifying the system, Eqn. (12)

\begin{tabular}{|c|c|c|c|}
\hline$P$ & $b_{1}$ & $b_{2}$ & $a$ \\
\hline 2 & 25.0 & 100.0 & 1 \\
\hline
\end{tabular}

Table 2

Parameters identifying the system, Eq. (17)

\begin{tabular}{|c|c|c|c|}
\hline $\mathrm{A}_{1}$ & $\mathrm{~A}_{2}$ & $\xi_{1}$ & $\xi_{2}$ \\
\hline 0.06666 & -0.06667 & -5.0 & -20.0 \\
\hline
\end{tabular}

The errors for different DTS's are shown in Table 3.

Table 3

Errors pertaining to discrete-time systems of different orders

\begin{tabular}{|l|c|c|c|c|}
\hline Order & First & Second & Third & Fourth \\
\hline Error & $0.254506 \mathrm{E}-02$ & $0.971892 \mathrm{E}-05$ & $0.234843 \mathrm{E}-04$ & $0.877769 \mathrm{E}-03$ \\
\hline
\end{tabular}

It is clear from Table 3 that the DTS of the second order is the system with minimum error. The parameters of the pertaining second order DTS are listed in Tables 4 and 5.

Table 4

Parameters identifying the second order discrete-time system, Eqn. (25)

\begin{tabular}{|l|c|c|c|}
\hline Parameter & $\beta_{1}$ & $\beta_{2}$ & $\alpha$ \\
\hline Value & $-0.1765611 \mathrm{E}+01$ & $0.7640908 \mathrm{E}+00$ & $0.996642 \mathrm{E}-04$ \\
\hline
\end{tabular}

Table 5

Parameters identifying the second order discrete-time system, Eqn. (26)

\begin{tabular}{|l|c|c|}
\hline Parameter & $\lambda_{1}$ & $\lambda_{2}$ \\
\hline Value & 0.95601 & 0.80010 \\
\hline
\end{tabular}

where $\lambda_{1}$ and $l_{2}$ are two roots of the characteristic equation of the corresponding DTS. From equations (26) and (28), the parameters $B_{2}$ and $B_{2}$ for the discrete-time system can be calculated as

$$
B_{1}=\frac{\alpha}{1-\lambda_{2} \lambda_{1}^{-1}}=6.111 \times 10^{-4} \quad ; \quad B_{2}=\frac{\alpha}{1-\lambda_{1} \lambda_{2}^{-1}}=-5.111 \times 10^{-4}
$$


Then, from Eqn. (33), the parameters for the continuous system can be easily obtained as

$$
\begin{aligned}
& A_{1}=\frac{1}{\Delta T} B_{1}=0.06111 \\
& A_{2}=\frac{1}{\Delta T} B_{2}=-0.5111 \\
& \xi_{1}=\ln \lambda \cdot \frac{1}{\Delta T}=-4.4987 \\
& \xi_{2}=\ln \lambda_{2} \cdot \frac{1}{\Delta T}=-22.302
\end{aligned}
$$

The estimated and exact values of the parameters of the corresponding continuous system are listed in Table 6.

\section{Table 6}

Estimated and exact values of the parameters of the continuous system

\begin{tabular}{|l|c|c|c|c|}
\hline Parameter & $\mathrm{A}_{1}$ & $\mathrm{~A}_{2}$ & $\xi_{1}$ & $\xi_{2}$ \\
\hline Exact & 0.066676 & -0.06667 & -5.0 & -20.0 \\
\hline Estimated & 0.06111 & -0.05111 & -4.4987 & -22.302 \\
\hline
\end{tabular}

Figure 2 shows the exact and estimated responses of the system using discrete time system of the $2^{\text {nd }}$ order DTS. Meanwhile, Figure 3 shows the exact and estimated system characteristic function $h(t)$ for the $2^{\text {nd }}$ order DTS.

\section{Example (2)}

Consider the system

$$
\dot{y}+5 y=100 \sin \left(0.5 t^{1.5}\right)
$$

where the input $x(t)$ is

$$
x(t)=100 \sin \left(0.5 t^{1.5}\right)
$$

The corresponding input discrete-time series is plotted in Fig. 1, with $\Delta \mathrm{T}=0.01$. The parameters of this system are listed in Table 7 below. 


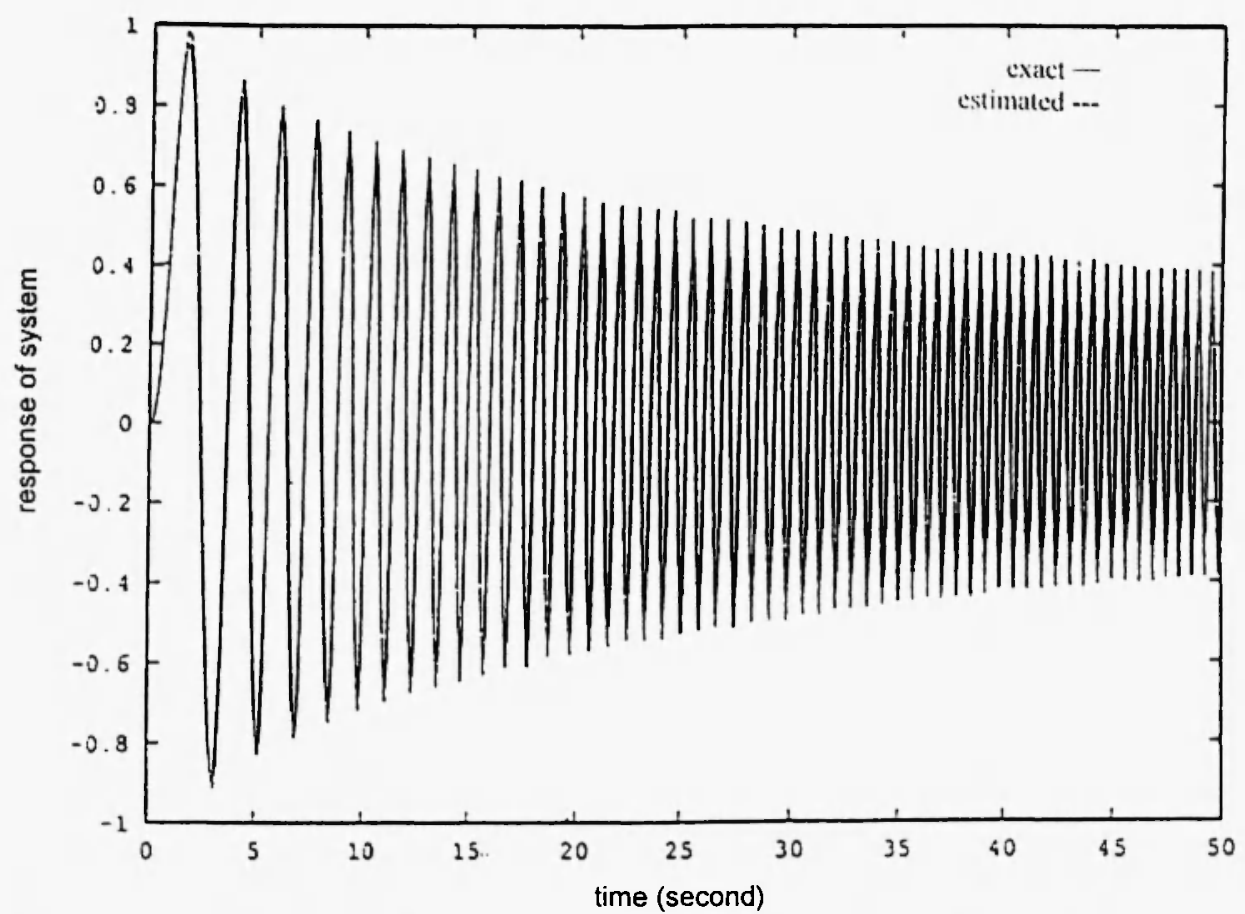

Fig. 2: The exact and estimated responses of the 2nd order DTS. Second order system: $\ddot{y}+25 \dot{y}+100 y=x(t)$ with parameters $\mathrm{a}=1.0, \mathrm{~b}_{1}=25, \mathrm{~b}_{2}=100, \mathrm{p}=2$ and input $\mathrm{x}(\mathrm{t})$ of Fig. 1 .

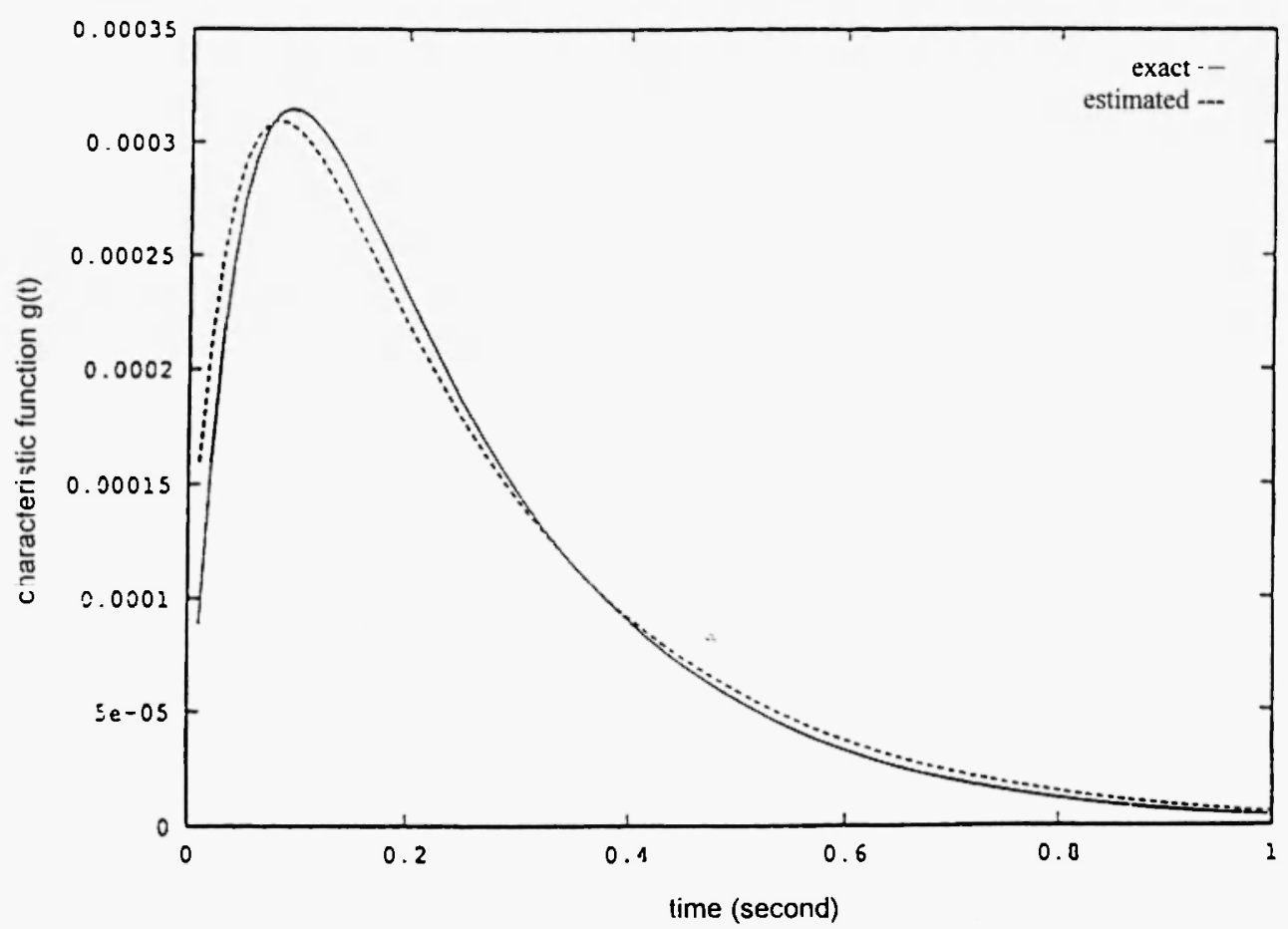

Fig. 3: The exact and the estimated system characteristic function $h(t)$ determined for the 2 nd order DTS. Second order system: $\ddot{y}+25 \dot{y}+100 y=x(t)$ with parameters $\mathrm{a}=1.0, \mathrm{~b}_{1}=25, \mathrm{~b}_{2}=100, \mathrm{p}=2$ and input $x(t)$ of Fig. 1 . 
Table 7

Parameters identifying the system, Eqn. (12)

\begin{tabular}{|c|c|c|c|c|}
\hline $\mathrm{p}$ & $\mathrm{b}_{1}$ & $\mathrm{a}$ & $\mathrm{A}_{1}$ & $\xi_{1}$ \\
\hline 1 & 0.5 & 1 & 1.0 & -5.0 \\
\hline
\end{tabular}

The errors for discrete-time systems of different orders are listed in Table 8 below.

Table 8

Errors for discrete-time systems of different orders.

\begin{tabular}{|c|c|c|c|}
\hline Order & First & Second & Third \\
\hline Error & $0.852409 \mathrm{E}-02$ & $0.253536 \mathrm{E}+00$ & $0.634591 \mathrm{E}-1$ \\
\hline
\end{tabular}

From Table 8, the DTS of the first order is the system with minimum error, the parameters of which are given in Table 9.

Table 9

Parameters identifying the first order discrete-time system, Equations (25) and (26)

\begin{tabular}{|c|c|c|c|}
\hline Parameter & $\beta$ & $\alpha$ & $\lambda$ \\
\hline Value & $-0.952681 \mathrm{E}+00$ & $0.951930 \mathrm{E}-02$ & 0.95268 \\
\hline
\end{tabular}

The estimated and exact values of the parameters of the continuous system model are listed in Table 10.

Table 10

Estimated and exact values of the parameters of the continuous system

\begin{tabular}{|c|c|c|}
\hline Parameter & $\mathrm{A}_{1}$ & $\xi_{1}$ \\
\hline Exact & 1.0 & -0.5 \\
\hline Estimated & 0.95193 & -4.8476 \\
\hline
\end{tabular}

Figure 4 shows the exact and the estimated responses given by the 1st order DTS. Figure 5 shows the exact and the estimated system characteristic functions $h(t)$ obtained for the first order DTS. 


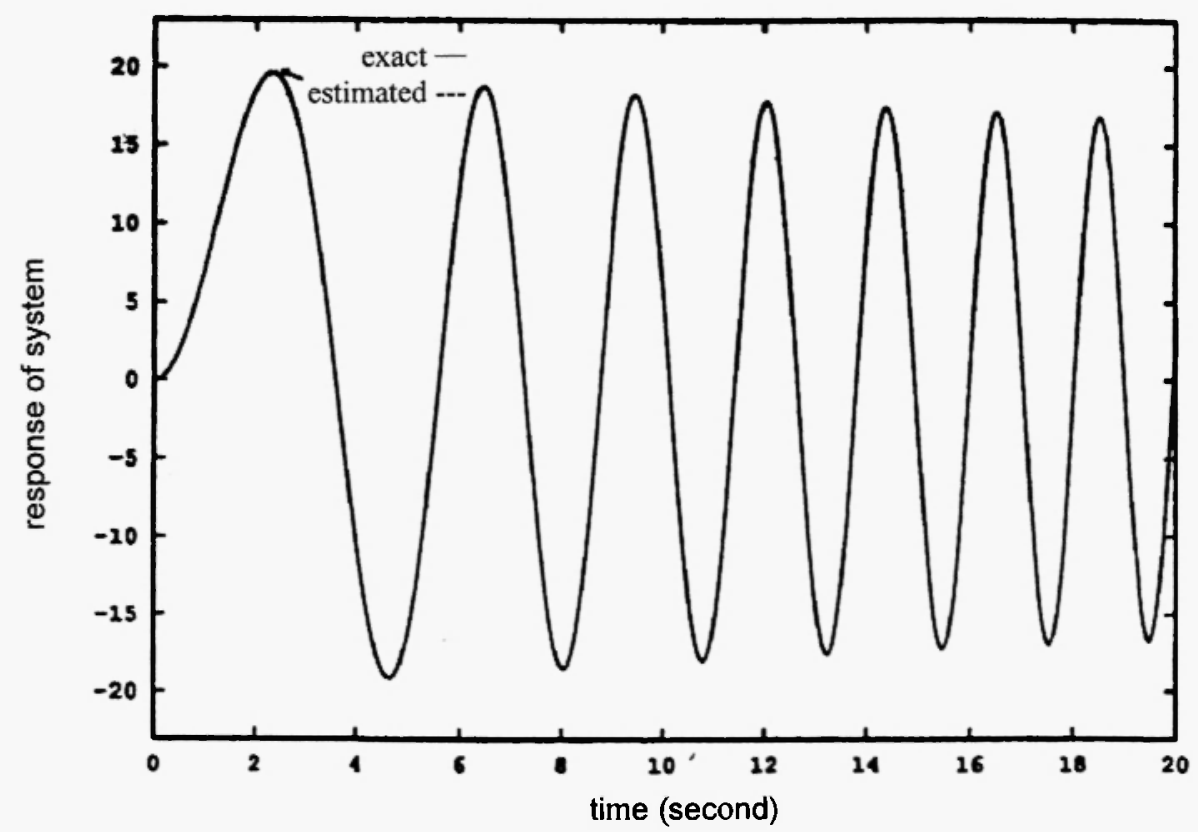

Fig. 4: The exact and the estimated responses of the 1st order DTS. First order system: $y+5 y=x(t)$ with parameters $a=1.0, b_{1}=0.5, p=1$ and input $x(t)$ of Fig. 1 .

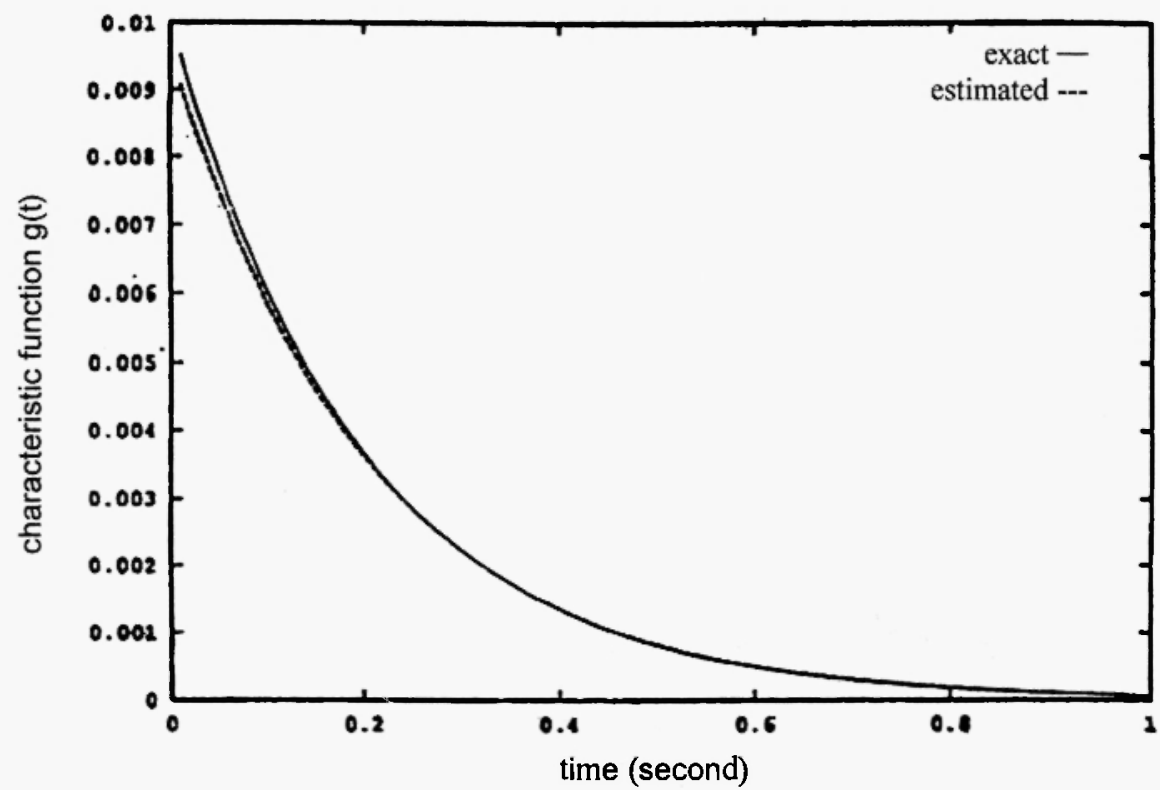

Fig. 5: The exact and the estimated system characteristic function $h(t)$ of the 1st order DTS. First order system $\dot{y}+5 y=x(t)$ with parameters $\mathrm{a}=1.0, \mathrm{~b}_{1}=0.5, \mathrm{p}=1$ and input $\mathrm{x}(\mathrm{t})$ of Fig. 1. 


\section{CONCLUSION}

A dynamic system identification method is proposed for the determination of the characteristic function of a class of linear rheological material systems by using dynamic signal processing and analysis. A model of rational function of polynomials for the pertaining transfer function is assumed. A discrete-time system analysis method is, first, introduced to identify the order and parameters of the model. Then, the characteristic function of the system is obtained via the inverse Laplace transform. A numerical analysis model and illustrative examples for testing this model are given. The results show that the proposed method is efficient and easy to use.

\section{REFERENCES}

Y.M. Haddad, Viscoelasticity of Engineering Materials, Kluwer, Dordrecht, 1995.

G. Fodor. Laplace Transforms in Engineering, Publishing House of the Hungarian Academy of Sciences, Budapest, 1965.

L.J. Morris, Computation Methods in Elementary Numerical Analysis, John Wiley \& Sons, New York, 1983.

P. Yu and Y.M. Haddad. A discrete-time dynamic system method for the identification of the viscoelastic properties of a class of materials, Int. J. Press. Ves. \& Piping, 62, 291-301 (1995). 
